\title{
A TATA-like sequence located downstream of the transcription initiation site is required for expression of an RNA polymerase II transcribed gene
}

\author{
Juan Carcamo, Edio Maldonado, Patricia Cortes, Moon-Hwan Ahn, Ilho-Ha, Yumi Kasai, ${ }^{1}$ Jane \\ Flint, ${ }^{1}$ and Danny Reinberg \\ Department of Biochemistry, Robert Wood Johnson Medical School, University of Medicine and Dentistry of New Jersey, \\ Piscataway, New Jersey 08854-5635 USA; ${ }^{1}$ Department of Molecular Biology, Princeton University, Princeton, New Jersey \\ 08544 USA
}

TFIID, the TATA-binding protein, was found to stimulate transcription from the adenovirus IVa2 promoter, a promoter considered to lack the TATA motif. Remarkably, a TATA-like sequence element located downstream of the transcription start site binds TFIID and is required for TFIID-dependent transcription from the IVa2 promoter. Transcription from the IVa2 and the adjacent adenovirus major late promoter (Ad-MLP) is divergent, and the cap sites are separated by 212 nucleotides. Nevertheless, the TATA motifs of the IVa2 promoter and Ad-MLP were found to be oriented in the same direction. An initiator motif around the transcription start site is located in the IVa2 promoter, and in contrast to the TATA motifs, the IVa2-initiator is in the opposite orientation with respect to the initiator of the Ad-MLP. A model is presented in which the polar nature of the initiator governs the direction of transcription. We propose that RNA polymerase II and accessory factors recognize the initiator in an orientation-dependent fashion. The recognition of the IVa2 initiator by RNA polymerase is enhanced by the binding of TFIID to the downstream TATA motif.

[Key Words: RNA polymerase II; Ad-MLP; TFIID; TATA motif]

Received April 4, 1990; revised version accepted June 28, 1990.

Class II promoters are composed of several elements, usually located upstream of the start site of transcription (cap site). An element found in most class II promoters, located at about position -30 , contains a sequence that reads TATA. This so-called "TATA box" element, in conjunction with the cap site, appears to position the start site of transcription (Breathnach and Chambon 1981). Upstream of the TATA box are other promoter elements that function as recognition sites for specific DNA-binding proteins that appear to modulate the level of gene expression (McKnight and Tiian 1987; Carcamo et al. 1989).

Initially, it was thought that most class II promoters contained a TATA sequence; however, another class of RNA polymerase II-transcribed genes, in which the TATA box element is absent from the promoter, has now been identified (Baker and Ziff 1981; Ayer and Dynan 1988; Moura-Neto et al. 1989; Smale and Baltimore 1989 and references therein). Many genes are included in this category, and a common alternate transcriptional control sequence has not yet been identified.

It has been demonstrated previously that transcription in vitro from different class II TATA-containing promoters required at least five different factors in addition to RNA polymerase II (for review, see Mermelstein et al. 1989; Saltzman and Weinmann 1989). These factors (TFIIA, TFIIB, TFIID, TFIIE and TFIIF) operate via minimal promoter sequences, the TATA box and the cap site, and are required to generate a stable complex between the RNA polymerase II and the promoter (Reinberg et al. 1987b; Buratowski et al. 1989). TFIID appears to be the only general transcription factor that contains a sequence-specific DNA-binding activity with specificity for the TATA motif (Davidson et al. 1983; Reinberg et al. 1987b; Nakajima et al. 1988). Current studies suggest that the binding of TFIID to the TATA motif provides the recognition signal for RNA polymerase II and the other general transcription factors, allowing their entry into the transcription cycle (Fire et al. 1984, Reinberg et al. 1987a, 1987b; VanDyke et al. 1988; Buratowski et al. 1989).

TFIID activity has also been detected in Saccharomyces cerevisiae and is capable of functionally replacing the mammalian counterpart in transcription from the adenovirus major late promoter (Ad-MLP) in a system reconstituted with mammalian transcription factors (Buratowski et al. 1988; Cavallini et al. 1988). Interestingly, although the mammalian and yeast factors are 
functionally equivalent, they appear to have different physical properties. The human TFIID (hTFIID) activity elutes from gel filtration columns performed in high salt and sediments through glycerol gradients with a molecular mass of $\sim 100 \mathrm{kD}$ (Samuels et al. 1982; Reinberg et al. 1987b), whereas the yeast TFIID activity (yTFIID) elutes from a gel filtration column with an apparent molecular mass of $\sim 27 \mathrm{kD}$ (Buratowski et al. 1988; Horikoshi et al. 1989b).

To gain insight into the molecular mechanisms of transcription from non-TATA sequence-containing promoters, we studied the adenovirus IVa2 promoter. The cap site of this promoter is located 210 nucleotides upstream of the Ad-MLP (Baker and Ziff 1981). Transcription from these two promoters uses different DNA strands. This arrangement of the IVa2 promoter and MLP offers the possibility that they may share upstream regulatory elements. Analysis of the sequences of the $\mathrm{IVa} 2$ promoter indicated that the -30 region (in which the TATA motif is usually located in other mammalian genes) is rich in GC residues. Furthermore, previous studies have indicated that there are at least three DNA elements that affect transcription from this TATA-less promoter. A proximal element is centered around position -30 , and another element overlaps the major late upstream promoter factor site [MLTF (Carthew et al. 1985), also known as USF (Sawadogo and Roeder 1985a) or UEF (Miyamoto et al. 1985)], both of which positively regulate transcription (Natarajan et al. 1984, 1987). The third element, which inhibits transcription, encompasses the TATA and cap sites of the Ad-MLP (Natarajan et al. 1984, 1985). We have demonstrated previously that purified MLTF stimulated transcription from the IVa2 promoter in a system reconstituted with partially purified general transcription factors (Carcamo et al. 1989) and, in addition, have documented that transcription from the IVa2 promoter required all of the previously described general transcription factors (Carcamo et al. 1989). In the studies presented here, we demonstrate that a TATA-like sequence located downstream of the cap site directs transcription of the IVa2 promoter in a TFIID-dependent fashion.

\section{Results}

The TATA-binding protein is required for transcription of the IVa2 promoter

It has been suggested that the binding of TFIID to the TATA sequence provides the recognition site for RNA polymerase II and other general factors, thereby allowing entry into the transcription cycle (Fire et al. 1984; Reinberg et al. 1987b; Horikoshi et al. 1988b; VanDyke et al. 1988; Buratowski et al. 1989). To isolate an equivalent factor required for transcription of the IVa2 promoter, we attempted to reconstitute transcription in vitro from this TATA-less promoter, using purified proteins. When saturating amounts of TFIIA, TFIIB, TFIIE, TFIIF, and RNA polymerase II, together with MLTF, were incubated with a plasmid DNA containing the IVa2 promoter, no transcription was observed /data not shown, see below $\mid$. However, the addition of limiting amounts of nuclear extract /a concentration that, by itself, will not catalyze transcription) resulted in transcription (data not shown). The activity present in the extract and complementing IVa2 transcription in the reconstitute assay described above copurified with TFIID.

Because the hTFIID activity has not been successfully purified to homogeneity and antibodies against this activity are not available, we could not unequivocally demonstrate that TFIID participated in transcription from the TATA-less promoter. Therefore, we analyzed further the requirement for TFIID in transcription from the IVa2 promoter by substituting the human activity with TFIID purified from yeast (yTFIID). The yTFIID gene has been cloned (Cavallini et al. 1989; Eisenmann et al. 1989; Hahn et al. 1989; Horikoshi et al. 1989a; Schmidt et al. 1989). Therefore, to conclusively analyze whether TFIID was required for transcription of the IVa2 promoter, a yTFIID clone that allows overexpression of yTFIID in Escherichia coli (gift of Dr. A. Berk) was used to purify the activity. The purification of yTFIID from $E$. coli is summarized in Figure 1A. The homogeneous protein, as expected, directed transcription from the AdMLP (Fig. 1B). In agreement with our previous results indicating that hTFIID was required for transcription of the IVa2 promoter (Carcamo et al. 1989), yTFIID isolated from $E$. coli was capable of directing transcription from the adenovirus IVa2 promoter, as indicated by the absence of detectable transcripts in reactions reconstituted with the general transcription factors but without TFIID (Fig. 1B, lane 3) and by the level of transcription proportional to the amount of $E$. coli-produced TFIID protein added to the reaction mixture (Fig. 1B, lanes $4-11$ ). The level of transcriptional activity with the bacteria-synthesized protein was similar to that observed with hTFIID and yTFIID (Fig. 1B, cf. lanes 1, 2, and 11). Thus, this result demonstrated that TFIID was required for transcription from this so-called TATA-less promoter.

TFIID directs transcription of the IVa2 promoter from the transcription start sites used in vivo

The cap sites of the adenovirus IVa2 promoter and MLP are separated by 212 nucleotides and are transcribed from opposite DNA strands. The area between the two cap sites is functionally complex (Yu and Manley 1986) and contains recognition sites for at least three different DNA-binding proteins, TFIID, MLTF, and NTF (nonTATA factor; J. Carcamo, M. Ahn, and I. Olave, in prep.; see Fig. 2A). Of particular interest to our studies is the sequence arrangement of the two TATA box elements surrounding the MLTF recognition site, especially the one located proximal to the IVa2 cap site (position -140 ; see Fig. 2A) because it is in the opposite orientation with respect to the MLP TATA box and thus could be directing transcription in the same direction as the transcripts originating from the IVa2 cap site. Therefore, we investigated whether TFIID-directed transcription from the IVa2 promoter originated at the cap site used in vivo. 
A

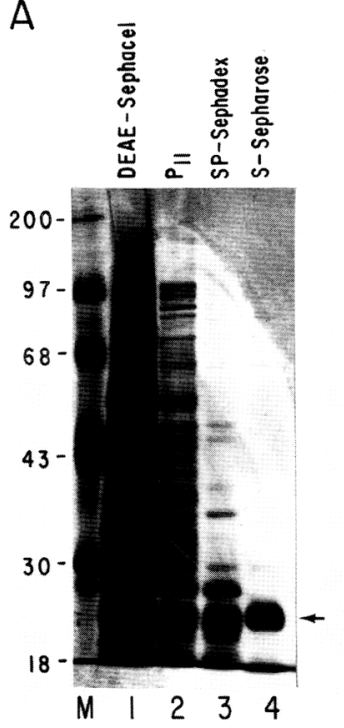

B

$$
\begin{aligned}
h I I D(\mu g) & 1.2 \\
Y I D(\mu g) & -3.1 \\
Y I I D(E . ~ c o l i)(n g) & --1.252 .5510204080160
\end{aligned}
$$
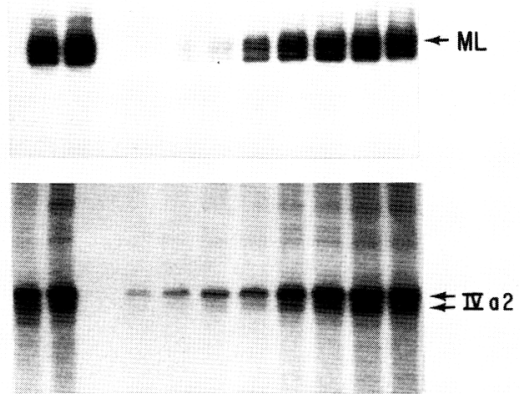

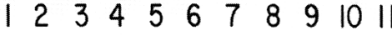

Figure 1. yTFIID produced in E. coli can substitute functionally for the hTFIID and yTFIID in transcription from the Ad-MLP and adenovirus IVa2 promoter. $(A)$ Silver staining of an SDS-polyacrylamide gel containing protein fractions of different steps of purification of yTFIID isolated from E. coli cells transformed with a plasmid DNA containing the yTFIID clone (gift of Dr. A. Berk) that allows overexpression. The purification procedure will be published elsewhere (E. Maldonado, P. Cortes, L. Weiss, and D. Reinberg, in press). (B) hTFILD (carboxymethyl cellulose fraction, lane 1), yTFIID (Superose-12 fraction, lane 2), or different amounts of the bacteria-produced yTFIID (S-Sepharose fraction; see $A$, lanes 4-11) were added, as indicated at top of $B$, to transcription reaction mixtures reconstituted with purified general transcription factors isolated from HeLa cells and containing the Ad-MLP or IVa2 promoter. Reaction conditions were as described in Materials and methods, and products were separated by electrophoresis.

In the studies described above, we used a runoff assay. The DNA template was linearized at a unique HincII recognition site located 656 nucleotides downstream of the IVa2 cap site. Thus, transcription initiating at the IVa2 cap site used in vivo should generate a transcript of 656 nucleotides (see Fig. 2A). However, if the TATA box located at position -140 were directing transcription, these transcripts would have to be $\sim 760$ nucleotides (transcription starting $\sim 30$ nucleotides downstream of the TATA motif). Analysis of the products generated by use of the runoff assay indicates the presence of transcripts of $\sim 650$ nucleotides (Fig. $2 B$, see arrow), in addition to transcripts larger than 1000 nucleotides that probably represent nonspecific initiation sites located in the vector sequences (Natarajan et al. 1984). Thus, these results suggest that transcription started primarily at or around the nucleotides of the cap site used in vivo. To map the start site of transcription precisely and also to analyze the activity of the different TFIID proteins (hTFIID and E. coli-produced yTFIID), reactions were performed and the products were analyzed by use of a primer extension assay. The primer used extended from +75 to +45 ; therefore, transcripts initiated at the major cap sites used in vivo should generate cDNAs of $\sim 78$ nucleotides (see Fig. 2A). When different amounts of hTFIID were added to IVa2 transcription reactions, and the products were analyzed as described above, a major band of $\sim 78$ nucleotides was observed in addition to minor start sites mapping around $-40,-60$, and -100 (Fig. 2C). The major transcript starting at +1 , as well as the minor transcripts, were dependent on TFIID and responded linearly with respect to protein concentration (cf. lanes 2-5 with 1). The same start sites were observed with yTFIID (Fig. 2C, lanes 6 and 7). Therefore, these results demonstrate that TFIID-dependent transcription from the IVa2 promoter is initiated in vitro primarily at the same site used in vivo. The transcripts initiating at "cryptic sites" were observed only in reactions reconstituted with the general transcription factors and were absent, or drastically decreased, when extracts and circular DNA were used (see below). The presence of transcripts starting at random sites on the DNA template is a common feature of the in vitro system; however, the fact that the most abundant transcript used the same site used in vivo and was dependent on TFIID suggests strongly that specific transcription from the IVa2 promoter requires TFIID.

\section{TFIID binds to a TATA-like sequence located downstream of the transcriptional start site}

In the studies presented above, we demonstrated that TFIID was required for transcription of the IVa2 promoter. We then investigated whether TFIID could recognize any DNA sequences in the IVa2 promoter. When different amounts of yTFIID were added to DNase I footprinting reactions containing the IVa2 promoter, protection was observed over sequences located downstream of the transcriptional start site and extended from residues +25 to approximately +10 , in addition to a weak protection to sequences located at -40 (Fig. 3 , cf. lane 1 with lanes $2-4)$. The TFIID-dependent protection was competed by an oligonucleotide that contained the Ad-MLP TATA motif (lanes 7 and 8) or by an oligonucleotide containing IVa2 sequences from +24 to -10 (lanes 5 and 6) but not by oligonucleotides containing recognition sites for other known transcription factors such as the cAMP response element (lanes 9 and 10), NF-I, Sp1, or AP-1 (data not shown); thus, the observed binding was due to TFIID.

Analysis of the DNA sequence located downstream of the IVa2 cap site revealed the presence of a TATA-like element $\left(5^{\prime}\right.$-TATAGAAA-3') located between residues +21 and +14 . When present in an oligonucleotide (IVa2 
A

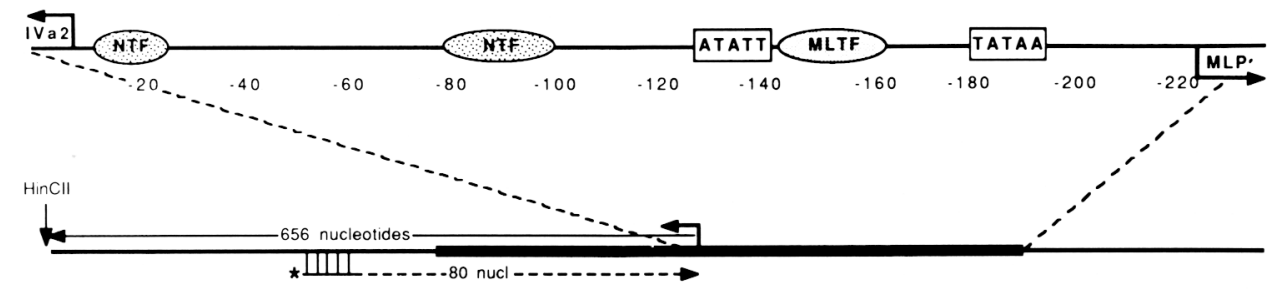

B

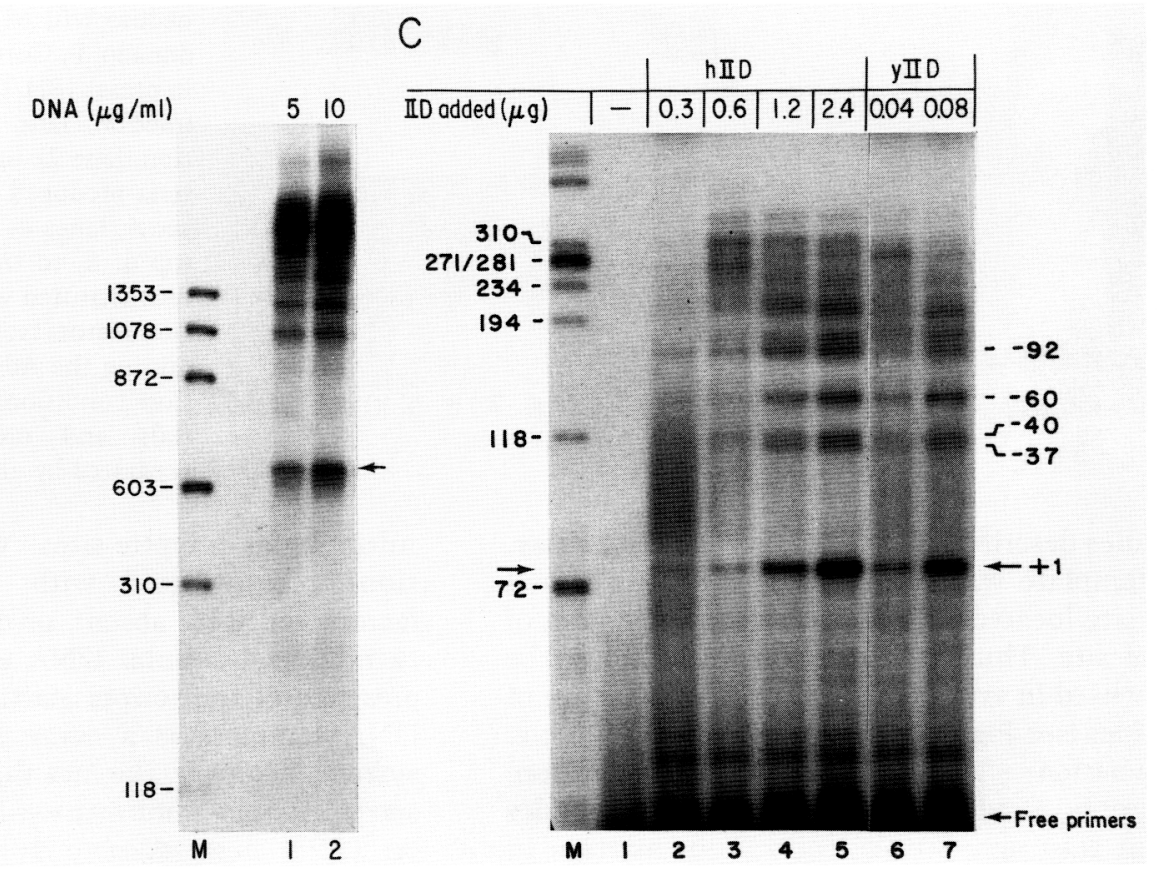

Figure 2. Analysis of the IVa2 transcriptional start site. (A) Schematic representation of the IVa2 promoter and MLP and the expected products from transcription reactions in vitro. The top line indicates sites present in the overlapping IVa2 promoter and MLP that serve as recognition for known specific DNA-binding proteins. (NTF) Non-TATA factor. The box closer to the MLP cap site, between residues -180 and -200 , represents the recognition site TFIID. The box closer to the IVa 2 cap site, between residues -120 and -140 next to the MLTF site, indicates another TATA sequence but in the inverted orientation with respect to the MLP TATA box. The arrow starting from the IVa2 cap site to the HincII site indicates the expected size of RNA molecules if they are correctly initiated at the IVa2 cap site when using a runoff assay. The arrow at bottom indicates the sizes of the expected products, when analyzed in a primer extension assay, if they are correctly initiated at the IVa2 cap site. The products of a runoff transcription assay with two different concentrations of DNA are shown in $B$. Reactions were performed as described in Materials and methods and contained plasmid DNA pAd-204, which contains IVa2 promoter sequences up to -204 , as indicated at top. The DNA was linearized with the restriction endonuclease HincII, as indicated in $A$. Lane $M$ contains ${ }^{32} \mathrm{P}-$ labeled DNA fragments $(\phi X 174, \mathrm{RF}$ DNA/HaeIII). The arrow at right indicates the specific transcripts originated from the IVa2 cap site. Primer extension analysis of the products obtained from transcription reactions reconstituted with purified factors is shown in $C$. Reaction mixtures received different amounts of hTFIID (Superose-12 fraction) or yTFIID (E. coli synthesized, S-Sepharose fraction, lanes 6 and 7), as indicated at top. The primer used for the reverse transcription is described in $A$. The products were separated in a $10 \%$ polyacrylamide-urea gel. (Lane $M$ ) $5^{\prime}-32 \mathrm{P}-\mathrm{labeled}$ $\phi X 174$, RF DNA markers digested with HaeIII endonuclease.

promoter sequences from +22 to +10 ), this TATA-like element effectively competed the binding of TFIID to the TATA motif present in the Ad-MLP (data not shown). Thus, these experiments indicated that the sequence 5'-TATAGAAA-3', located downstream of the IVa2 cap site, was capable of affecting the binding of TFIID to the MLP TATA sequence and, therefore, strongly suggested that this sequence was responsible for directing transcription from the IVa2 cap site in a TFIID-dependent fashion.
The protection observed on the IVa2 promoter in the -40 region appears to be due to changes on the DNA, probably induced by the binding of TFIID to the downstream TATA-like motif. The -40 region does not include any TATA-like sequence, it is rich in GC residues, and it did not compete the binding of TFIID to the downstream sequence; furthermore, when an oligonucleotide extending from -20 to -50 was subcloned into a pBR322-derived vector, no binding of TFIID could be demonstrated to this region (data not shown). 


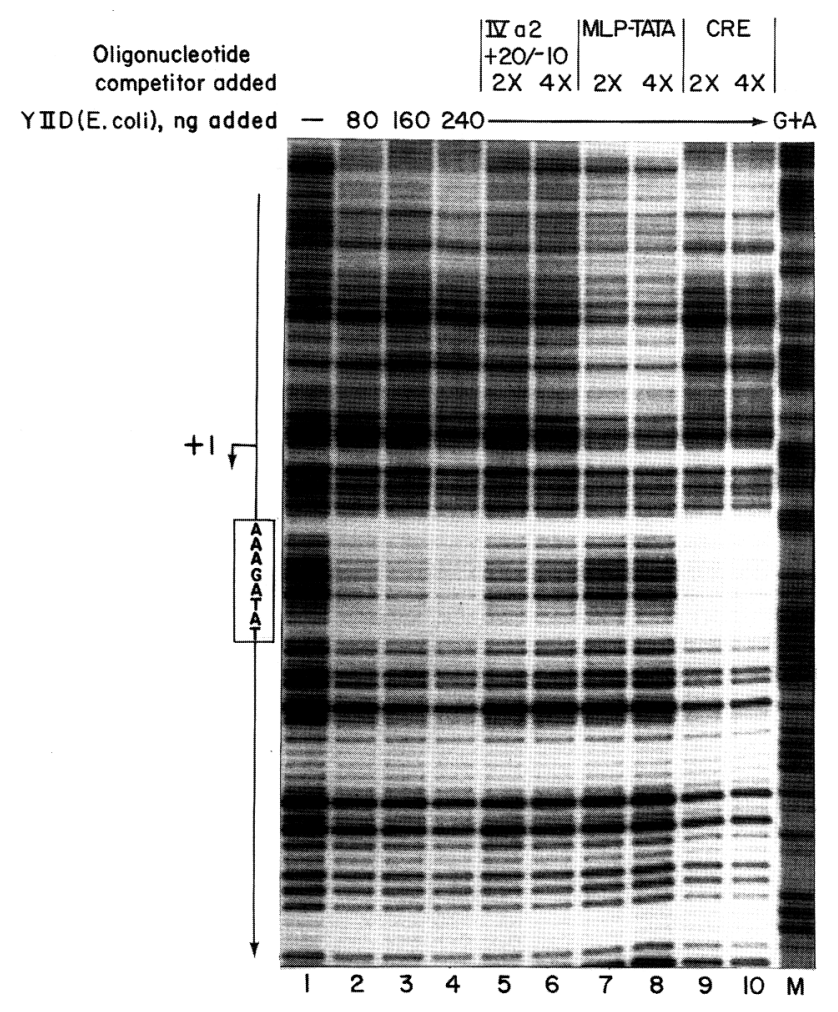

Figure 3. DNase I footprinting analysis of y'TFIID binding to IVa2 promoter sequences. Different amounts of yTFIID (E. coli synthesized, S-Sepharose fraction), as indicated at top, were added to DNase I footprinting reactions containing a DNA fragment that spanned the IVa2 promoter and MLP. The DNA fragment was isolated from plasmid DNA pDR31 (J. Carcamo, M. Ahn, and D. Reinberg, unpubl.) by double digestion with NheI and HindIII endonucleases. The DNA was $3^{\prime}$-end-labeled at the NheI site. DNase I footprinting reactions were carried out as described. The reaction mixtures also received different competitor oligonucleotides, as indicated at top. The samples were separated on $8 \%$ polyacrylamide-urea gels. The control pattern of DNase I digestion obtained in the absence of protein is indicated with a negative symbol $(-1)$ The relative positions of regions protected from DNase I in the IVa2 TATA-like element are indicated in an open box (left). The cap site of the IVa2 promoter is indicated at left. Maxam and Gilbert G + A markers are indicated as $M$ at bottom.

\section{The TATA-like sequence 5'-TATAGAAA-3', located downstream of the IVa2 cap site, is important for transcription from the IVa2 promoter}

The data described thus far indicated that TFIID was required for transcription from the IVa2 promoter and that TFIID was capable of recognizing sequences located downstream of the cap site. Furthermore, we found that a TATA-like sequence located at position +21 to +14 was capable of competing the binding of TFIID to the TATA motif present in the Ad-MLP. Together, these observations strongly suggested that this TATA-like sequence could be important for transcription from the IVa2 promoter. To analyze the importance of the downstream TATA motif on transcription from the IVa2 pro- moter, this sequence was mutated. Two mutants in this motif were generated by site-directed mutagenesis. We analyzed whether the level of transcription from the IVa2 promoter would increase if the TATA-like sequence was converted to a more conserved TATA motif such as the one present in the Ad-MLP, which is one of the strongest promoters studied thus far. In the second mutant, the TATA-like sequence was destroyed (for details, see Fig. 4A). The resulting DNA molecules were analyzed for their ability to bind TFIID and to drive transcription in nuclear extracts as well as in a TFIIDdependent fashion by use of a reconstituted system. Transcription from the DNA construct containing the Ad-MLP-TATA motif was approximately threefold more active than the wild-type construct (Fig. 4B, cf. lanes $8-10$ with $2-4$, respectively). This increase in activity correlated with an increased binding of TFID to the TATA motif (Fig. 5, cf. lanes 18-20 with 22-24). The substitution of the TATA motif by random nucleotides resulted in a construct (IVa2-TATA-Mut) that failed to support binding of TFIID (Fig. 5, see lanes 6-8) and had a reduced level of transcription (Fig. 4B, cf. lanes 5-7 with 2-4). The difference in the levels of transcription observed with these constructs reflects the importance of the TATA motif on transcription from the IVa2 promoter, as transcription from the Ad-MLP, which was included in the same reaction mixtures but on a separate DNA molecule as an internal control, was not affected (see bottom on Fig. 4B). To analyze further the effect of mutating the downstream TATA motif on transcription from the IVa2 promoter, the products of reactions containing the different TATA mutations were analyzed after 30 and 180 min of incubation. Consistent with the results presented in Figure 5, indicating better binding of TFIID to a more conserved TATA motif, the construct containing the Ad-MLP-TATA motif was transcribed more efficiently than the wild-type construct during the rate of the reaction (30 min of incubation; Fig. 4C, cf. lanes 10-12 with 2-4, respectively). On the other hand, when the transcription template activity of a construct containing no downstream TATA motif was compared to the wild-type template after $3 \mathrm{hr}$ of incubation, a more severe effect of this mutation was observed (Fig. $4 \mathrm{D}$, cf. lanes $3-5$ with $6-8$, respectively). Thus, these experiments demonstrate that the TATA-like sequence located downstream of the cap site is functional and that a more conserved TATA motif, such as the one present in the Ad-MLP, can also affect transcription from the IVa2 promoter when located downstream of the transcriptional start site.

Interestingly, when the transcription template activity of the construct containing a mutation in the TATA motif, which abolished binding of TFIID, was analyzed in a reconstituted system, transcription was dependent on TFIID (Fig. 6). The levels of transcription from the mutated template, in response to TFIID, were lower than those observed with the wild-type IVa2 construct (cf. lane 8 with 4 , respectively). The most logical interpretation of these results is that TFIID activity is enhanced by the TATA box element, as mutations that 
eliminate this motif resulted in lower levels of activity and mutations that yielded a consensus TATA box element resulted in an increase in TFIID binding that correlated with a higher level of transcription (Figs. 4 and 5). However, these results also suggest that TFIID participates in transcription independently of the TATA motif. This is in agreement with results indicating that transcription from an Ad-MLP construct containing a deletion of the TATA motif, or from the human $\beta$-DNA polymerase gene, which lacked the TATA motif (Widen et al. 1988) required TFIID (L. Buckbinder, E. Mal- donado, and D. Reinberg, in prep.; L. Weis and D. Reinberg, unpubl., respectively|. The most logical interpretation of these results is that TFIID can function, albeit at lower levels, independent of the TATA motif, probably by interacting with other transcription factors.

What defines the direction of transcription from the IVa2 promoter?

The results presented above demonstrated that a TATA motif located downstream of the IVa2 transcriptional
A

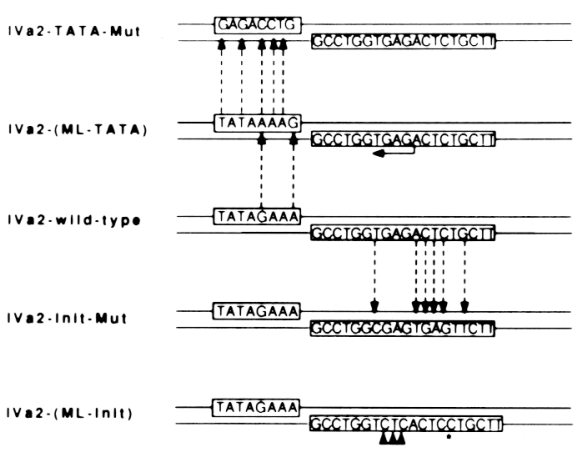

C

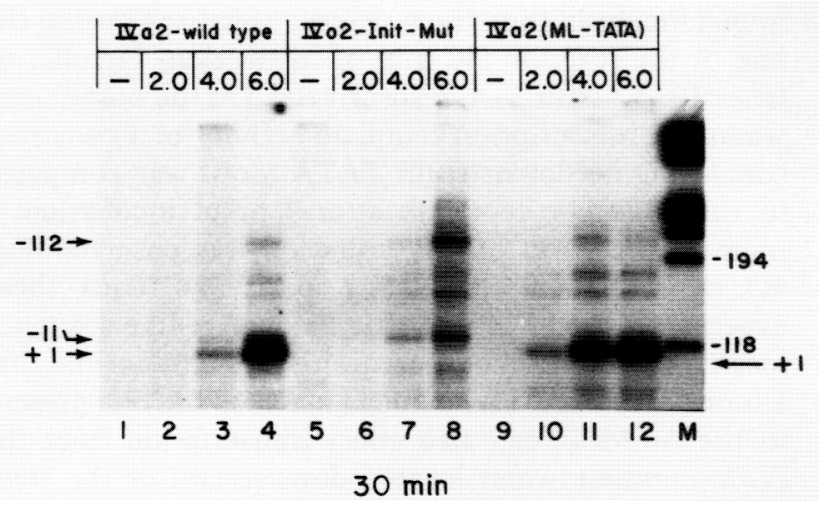

B

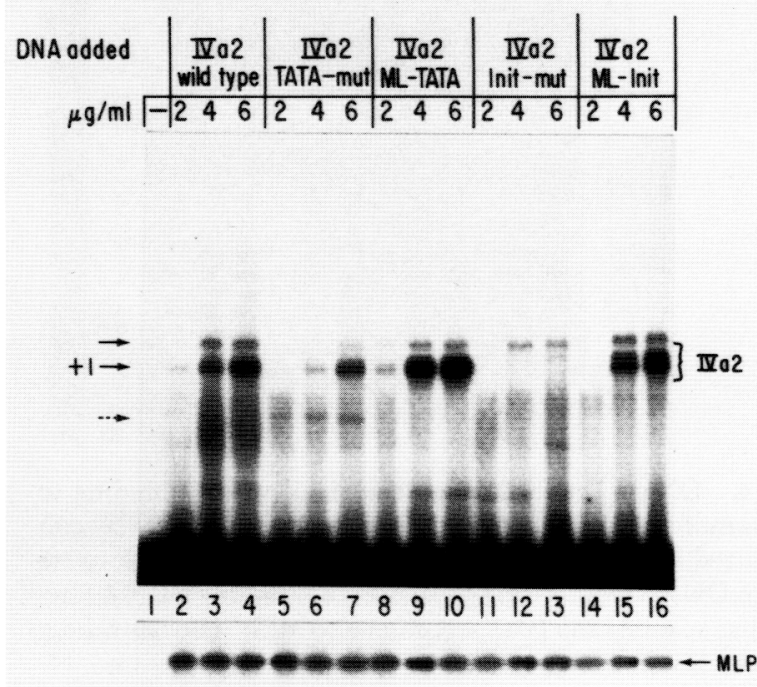

D

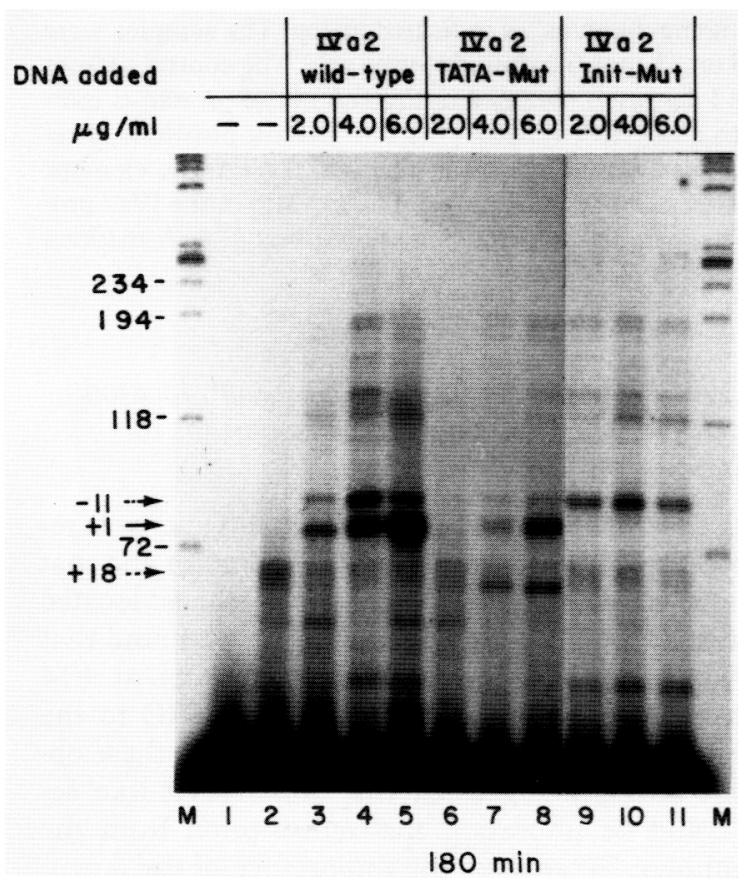

Figure 4. (See facing page for legend.) 
start site is important for transcription. Previous studies have indicated that the binding of TFIID to the TATA motif provides the recognition site for RNA polymerase II and the general transcription factors (TFIIB, TFIIE, TFIIF). Furthermore, these studies also indicated that the binding of the transcription complex was strictly unidirectional, forming downstream of the TATA motif and spanning the transcriptional start site (Horikoshi et al. 1988a,b; VanDyke et al. 1988). Interestingly, the TATA motif of the IVa2 promoter is located in the same orientation as the TATA motif of the Ad-MLP, which is $\sim 180$ nucleotides upstream from the IVa2 transcriptional start site. Nonetheless, transcription from these promoters is divergent (for details, see Fig. 8, below). Therefore, cis-acting elements other than the TATA motif must be responsible for orienting the direction of transcription. Analysis of the sequences within the IVa2 promoter indicates the presence of an element that shares homology with the "initiator" (Smale and Baltimore 1989) present in the promoter of the terminal deoxynucleotidyl transferase gene, a TATA-less promoter, as well as in the Ad-MLP (Fig. 7). Interestingly, the initiator motifs present in the IVa2 promoter and MLP are oriented in opposite directions (see Fig. 8A). Therefore, we analyzed the contribution of this element in transcription of the IVa2 promoter. Two independent mutants were generated by site-directed mutagenesis. In one mutant, the conserved nucleotides among the initiator present in the IVa2 promoter, MLP, and TdT promoter (see Fig. 7) were changed as indicated in Figure $4 \mathrm{~A}$. In the second mutant, nucleotides in the IVa2 initiator were changed to resemble the MLP initiator (see Fig. 4A). The transcriptional template activity of these constructs demonstrated that alteration of the conserved nucleotides in the initiator had a deleterious effect on specific initiation of transcription from the IVa2 promoter; however, initiation from a cryptic site upstream of +1 was not affected (Fig. 4B,C,D, see lanes 11-13, $6-8$, and 9-11, respectively). On the other hand, when the initiator from the IVa2 promoter was changed to that present in the MLP, transcriptional levels approximated those observed with the wild-type template (Fig. 4B, cf. lanes 14-16 with 2-4). In no case was transcription from an internal control, the Ad-MLP, affected (see bottom on Fig. 4B). Also these two mutations had no effect on the binding of TFIID to the downstream TATA element (Fig. 5, see lanes 9-16). Therefore, the results of these experiments demonstrate that the initiator motif is necessary for transcription of the IVa2 promoter.

It is pertinent to postulate that the initiator motif is recognized by at least one element of the transcription machinery, perhaps RNA polymerase II. Furthermore, because of the arrangement of the initiator present in the IVa2 promoter and MLP and its critical effect on transcription, it is pertinent to postulate that this element fixes the direction of transcription.

\section{Discussion}

In efforts to understand the DNA elements and protein factors regulating transcription from class II TATA-less promoters, we analyzed the adenovirus-encoded IVa2 promoter, a promoter referred to as TATA-less (Baker and Ziff 1981; Leong and Flint 1984; Natarajan et al. 1984; Carcamo et al. 1989). Our initial studies, with a functional transcription assay, suggested that transcription from this promoter required TFIID /Carcamo et al. 1989|, a general transcription factor known to contain a DNA-binding activity with specificity for the TATA motif (Nakajima et al. 1988). By analyzing the activities of the yTFIID and hTFIID proteins, in the studies described here, we demonstrated the involvement of TFIID in transcription from the IVa2 promoter. The involvement of the TATA-binding protein on transcription from the IVa2 promoter was demonstrated by isolation of TFIID from $E$. coli cells transformed with a plasmid DNA containing the yTFIID gene, which allows overproduction of the protein. The involvement of TFIID on

Figure 4. Transcription template activity of IVa2 promoter constructs with mutations in the TATA or initiator motifs. (A) Schematic representation of the different mutations introduced into the IVa2-TATA or initiator motifs. The boxes indicate the TATA and initiator motifs. The arrows originating from these boxes (broken lines) indicate the different mutations and the parental template used. The horizontal arrow on the IVa2-(ML-TATA) construct denotes the transcriptional start site, as well as the direction of transcription. The arrowheads on the IVa2-(ML-Init) construct indicate the nucleotide changes introduced into the IVa2 initiator motif. The asterisk $\left({ }^{*}\right)$ indicates the insertion of a $C$ residue into the IVa2 initiator motif. $(B)$ The transcriptional template activity of the constructs shown in $A$. Reaction mixtures were as described in Materials and methods and contained HeLa cell nuclear extracts, as well as variable amounts of the constructs shown in $A$, as indicated at top of $B$. Reaction mixtures also contained a plasmid DNA (2 $\mu \mathrm{g} / \mathrm{ml}$ ) with the Ad-MLP (pD-139 DNA; Reinberg and Roeder 1987). At the end of the incubation, reaction mixtures were divided in two. One reaction was used to analyze transcription from the IVa2 promoter, and the other was used for transcription of the Ad-MLP. The products of the reactions were analyzed by primer extension, as described in Materials and methods. The primer used to analyze +1 initiation from the IVa2 promoter was 30 nucleotides long and complementary to sequences extending from +78 to +48 . The +1 initiation is indicated at left. The solid arrow above +1 denotes a cryptic initiation site mapping at -18 . The broken arrow denotes a cryptic transcription initiation site at +22 . $(C)$ The transcriptional template activity of some of the constructs shown in $A$, as indicated at top of $C$. Reaction mixtures were as described in Materials and methods but incubated for 30 min and contained HeLa cell nuclear extracts as well as variable amounts of the construct DNAs, as indicated at top. The products of the reactions were analyzed by primer extension, as described in Materials and methods. The primer used to analyze +1 initiation from the IVa2 promoter was 30 nucleotides long and complementary to sequences extending from +115 to +85 . (D) The transcriptional template activity of the constructs shown in $A$, as indicated at top of $D$. Reaction mixtures were as described in Materials and methods but incubated for 3 hr and contained HeLa cell nuclear extracts as well as variable amounts of the constructs shown in $A$, as indicated at top of $D$. The products of the reactions were analyzed by primer extension, as described in Materials and methods. The primer used to analyze +1 initiation from the IVa2 promoter was 30 nucleotides long and complementary to sequences extending from +78 to +48 . 

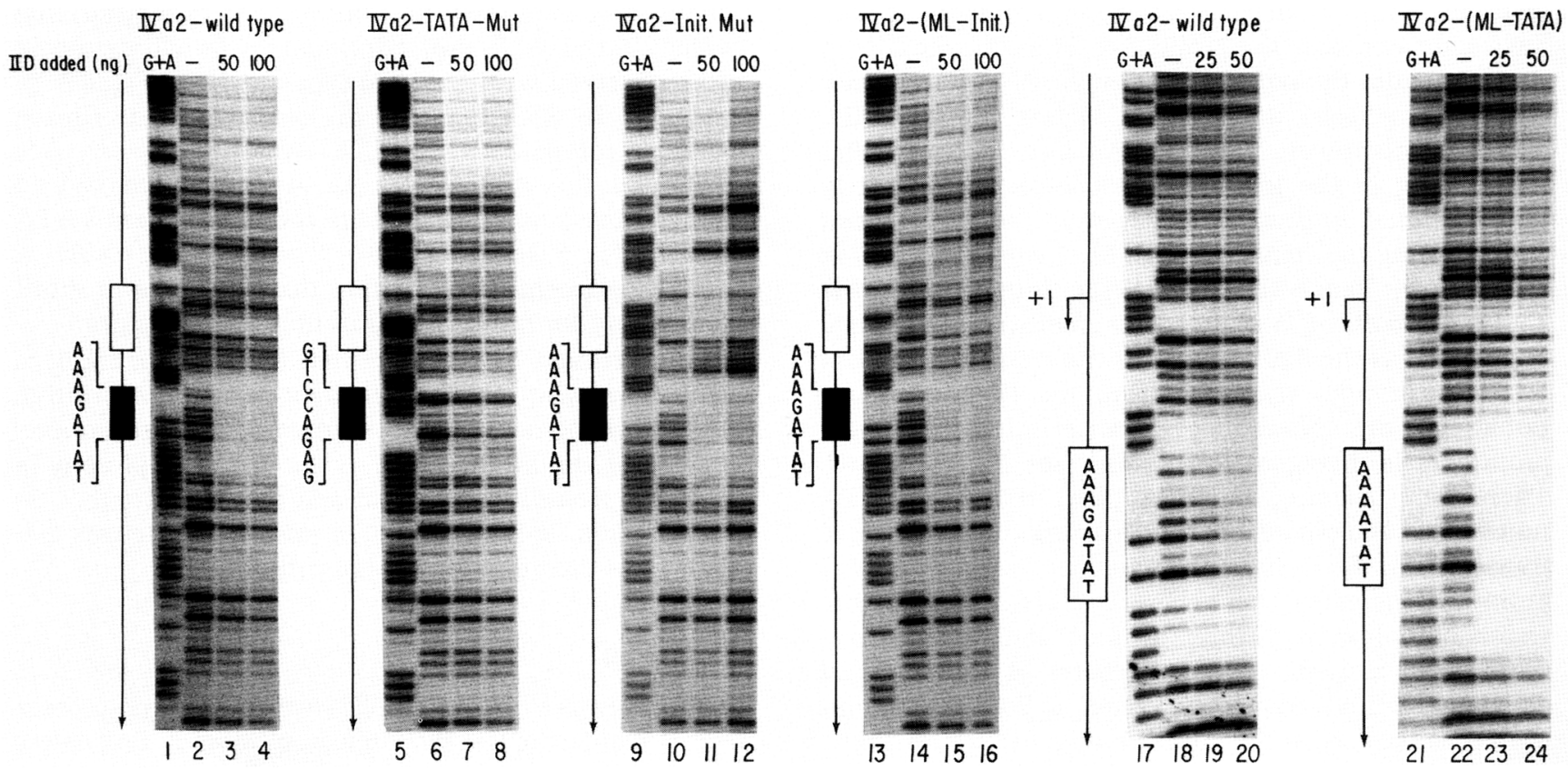

Figure 5. Analysis of the binding of TFIID to IVa2 promoter constructs containing different mutations in the TATA motif. The binding of yTFIID (E. coli-synthesized, S-Sepharose fraction) to the different IVa2 promoter constructs was analyzed by DNase I footprinting. The amount of TFIID added to the mixtures is indicated at top. The initiator and TATA-like elements are indicated as open and solid boxes, respectively (lanes $1-16$ ).

transcription from the IVa2 promoter is supported further by the finding that a sequence located downstream of the IVa2 transcriptional start site was capable of binding TFIID as well as competing the binding of TFIID to the TATA motif present in the Ad-MLP.

The DNA elements regulating transcription from the IVa2 promoter appear to be complex. Natarajan et al. $(1984,1985)$ have mapped three DNA elements that affected transcription. Two elements, one located between -50 and -30 , and another located between -150 and -180 , positively regulated expression. We have demonstrated previously that the distal element overlapped the MLTF recognition site and that this factor stimulated transcription from the IVa2 promoter (Carcamo et al. 1989). It was also demonstrated that sequences overlapping the Ad-MLP-TATA motif and cap site negatively regulated transcription from the IVa2 promoter (Natarajan et al. 1985). This latter result can be explained by the requirement of a common factor(s) for transcription from both promoters; however, because the IVa2 promoter is weak and the MLP is one of the strongest analyzed thus far, this factor(s) was sequestered by the stronger promoter. This is consistent with our observations demonstrating that TFIID was required for transcription from both promoters. The participation of TFIID in transcription from the IVa2 promoter is supported further by experiments demonstrating that either point mutations in the MLP TATA sequence or deletion of the MLP cap site, which drastically decreased or abolished transcription from the MLP, resulted in a relief of the negative effect on the IVa2 promoter (Natarajan et al. 1985).
In the studies presented here, we discovered that an element located downstream of the cap site positively affected transcription from the IVa2 promoter. Similar results have been obtained, particularly in the study of transcription from TATA-less promoters such as the SV40 late promoter (Ayer and Dynan 1988), the mammalian deoxynucleotidyl transferase gene (Smale and Baltimore 1989|, and the gene encoding the ribosomal protein L32 (Moura-Neto et al. 1989), among others. Although the IVa2 promoter has been classified as a nonTATA sequence-containing promoter (Baker and Ziff 1981; Leong and Flint 1984; Natarajan et al. 1984; Carcamo et al. 1989|, the studies presented here demonstrate that a TATA-like sequence, 5'-TATAGAAA-3' was located downstream of the transcriptional start site. This DNA element provided a recognition site for the TATA-binding protein and affected transcription, because mutation of this motif resulted in the elimination of TFIID binding and a decrease in the levels of transcription. Also, our experiments demonstrated that a conserved TATA motif such as the one present in the Ad-MLP $\left(5^{\prime}\right.$-TATAAAA- $\left.3^{\prime}\right)$ can substitute functionally for the IVa2 TATA-like sequence (5'-TATAGAAA- $\left.3^{\prime}\right)$; this substitution resulted in an increase in TFIID binding to the IVa2 promoter with a concomitant increase in the level of transcription from the IVa2 promoter.

The binding of TFIID to the Ad-MLP TATA sequence resulted in an area protected from DNase I cleavage that included the TATA motif and sequences located downstream toward the MLP cap site (Nakajima et al. 1988; VanDyke et al. 1988; Buratowski et al. 1989). However, 


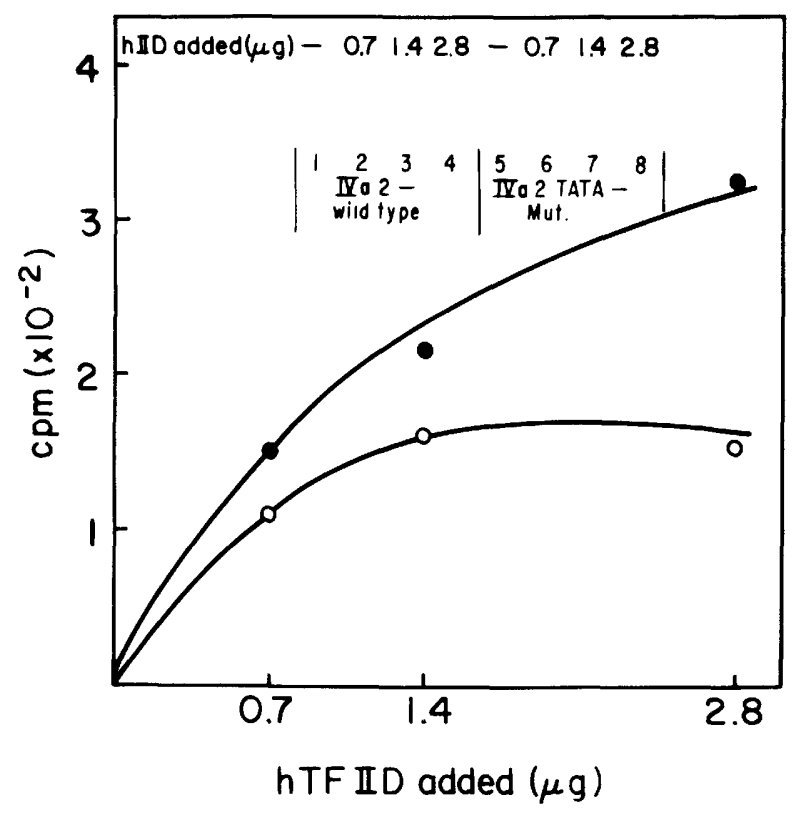

Figure 6. Effect of TFIID on transcription from IVa2 wild-type and IVa2-TATA-Mut promoter constructs. Reaction mixtures contained the general transcription factors TFIIA, TFIIB, TFIIE, TFIIF, RNA polymerase II, and MLTF, as indicated in Materials and methods. Reaction mixtures contained different amounts of hTFIID, as indicated. Products of the reactions were analyzed by primer extension, as described, and the amount of radioactivity incorporated into the specific transcript was determined by excision of the bands from the dry gel and counting. The extent of transcription from the IVa2-wild-type and IVa2TATA-Mut promoters, with respect to the amount of hTFIID added, are indicated as solid and open circles, respectively.

the binding of TFIID to the IVa2 promoter resulted in the protection of the TATA motif and upstream sequences, toward the IVa2 cap site. Studies with the adenovirus EIV promoter and MLP have demonstrated that the binding of TFIID allows RNA polymerase II and associated proteins to bind to promoter sequences. This binding is observed as an extension of the TFIID-protected area and extends over the cap site and downstream sequences. This is unlikely to happen on the IVa2 promoter, as transcription would then be directed toward the Ad-MLP (see Fig. 8). Therefore, elements unique to the IVa2 promoter must direct the binding of the polymerase to the transcribed DNA strand. Interestingly, Smale and Baltimore (1989) described a new transcriptional control element, the initiator. This DNA element is present in the promoter of the terminal deoxynucleotidyl transferase gene, a TATA-less promoter, as well as in the Ad-MLP. The initiator itself contains the transcriptional start site(s). Analysis of the sequences within the IVa2 cap site revealed homology with the initiator elements present in the TdT promoter and AdMLP (see Fig. 7). Interestingly, this DNA element is present in the nontranscribed DNA strand (see Fig. 8). Therefore, one could postulate that RNA polymerase II recognizes the initiator and binds to the DNA and that this binding must have directionality to allow transcrip- tion to proceed in a $5^{\prime} \rightarrow 3^{\prime}$ direction. Thus, because the initiators in the IVa2 promoter and MLP are located in different DNA strands (see Fig. 8), we postulate that RNA polymerase II recognizes the initiator in an orientation-dependent fashion. This recognition is greatly enhanced by, but not dependent on, the binding of TFIID to the TATA motif. It is possible that the binding of TFIID on the IVa2 promoter sequences opens the DNA strand and exposes the initiator motif to the polymerase. This is in agreement with our results indicating that mutations of the initiator eliminated transcription from the IVa2 promoter but mutations of the IVa2 TATA-like sequence resulted in a decrease in the level of transcription; however, the mutated promoter was capable of supporting low levels of transcription, and, more importantly, the start sites of transcription were not changed (see Fig. 4). These results are in agreement with the results of Smale and Baltimore (1989), indicating that a TATA box enhanced the levels of transcription within the initiator.

The experiments described here provide the first demonstration that a TATA sequence can be located downstream of the cap site and still direct transcription. Furthermore, these studies also suggest a functional role for the initiator motif in establishing the direction of transcription. The understanding of the molecular mechanisms by which the general transcription factors interact with the TATA sequence and the initiator present in the IVa2 promoter will provide insight into the mechanisms by which the general transcription factors interact with themselves and promoter sequences to initiate transcription.

\section{Materials and methods}

\section{Plasmid DNA}

The construction of the recombinant DNA molecules containing the Ad-MLP, pML(C2AT), was described previously by Sawadogo and Roeder (1985b). The recombinant DNA molecule containing the adenovirus IVa2 promoter (pAd-204) was described by Natarajan et al. (1984). This DNA molecule contains a deletion of sequences upstream of -204 , relative to the IVa2 cap site, and resulted in the removal of the MLP cap site. Plasmid DNAs containing point mutations in the IVa2 TATA [IVa2-TATA-Mut and IVa2-(ML-TATA)] or initiator [IVa2-InitMut and IVa2-(ML-Init)] motifs were produced by oligonucleo-

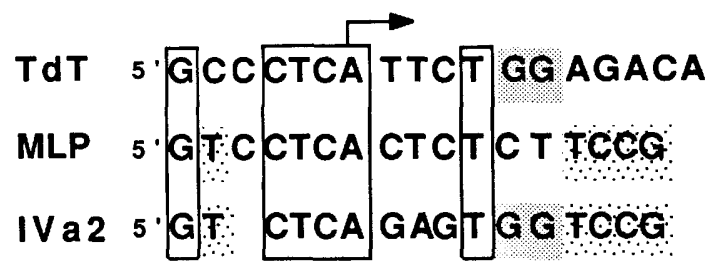

Figure 7. Similarity of the sequences of the initiator DNA elements present in the TdT promoter, MLP, and IVa2 promoter. The boxes indicate the nucleotide sequences that are conserved among the three initiators analyzed. The dark and light shadowed nucleotides indicate additional homologous regions between the IVa2 initiator with TdT and MLP initiators, respectively. The arrow at the top of the box represents the major transcription start site. 


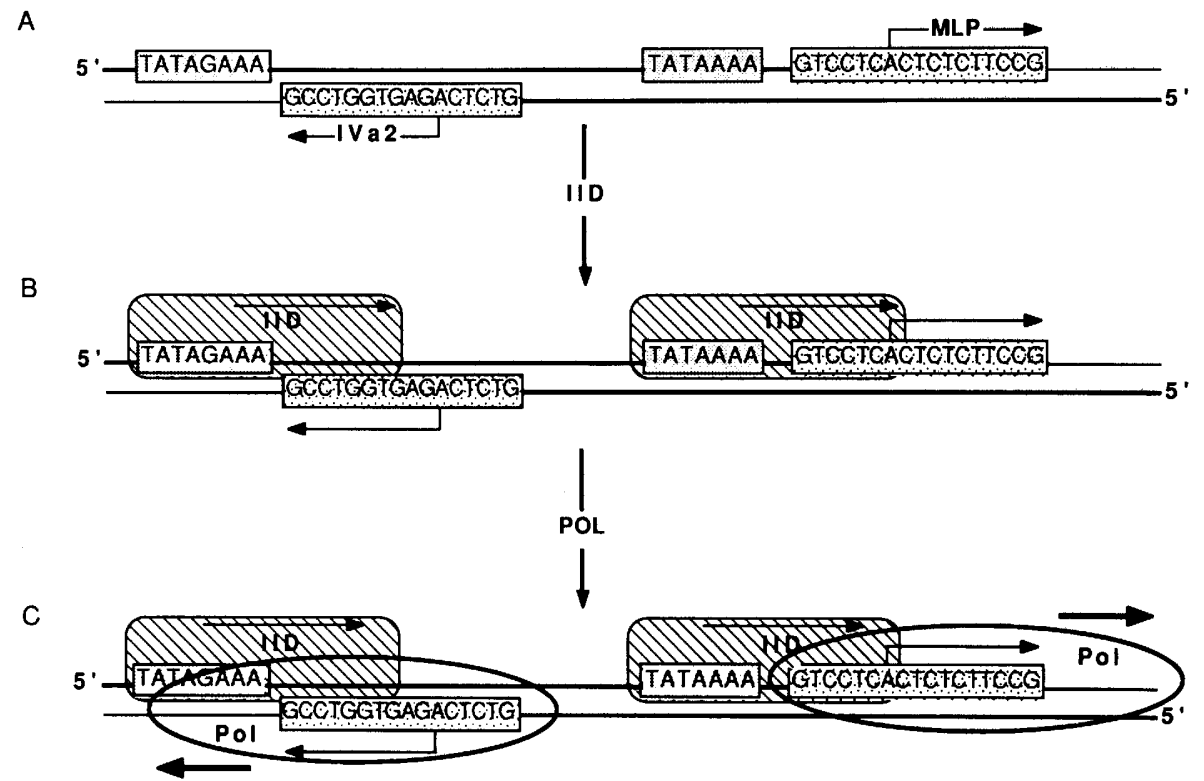

Figure 8. Model for the binding of RNA polymerase II and general transcription factors to the IVa2 promoter. $(A)$ Schematic representation of the cap site and TATA element present in the IVa2 promoter and MLP. The TATA-like sequence 5' -TATAGAAA-3' present in the IVa2 promoter is located downstream of the IVa2 cap site but in the same direction as the TATA sequence present in the Ad-MLP. The cap sites of the IVa2 promoter and MLP, as well as the directions of transcription from each, are indicated by an arrow. The boxes from where the arrows originate represent the initiator DNA elements. The thinner lines indicate the nontranscribed DNA strands. $(B)$ A schematic representation of the binding of TFIID to the IVa2 promoter and MLP-TATA motifs and surrounding sequences. The arrows inside the TFID boxes indicate the direction of binding of TFIID to the promoter sequences. It is possible that TFIID binds preferentially to the nontranscribed DNA strand. Binding to the transcribed DNA strand is indicated only for simplicity. All others are as described in $A$. (C) A schematic representation of the binding of RNA polymerase II to the IVa2 promoter and MLP. The polymerase binds downstream of TFIID on the MLP, whereas on the IVa2 promoter, the RNA polymerase II binds toward the TATA sequence. It is postulated that the polymerase recognizes the initiator DNA element, which is indicated by the box. This DNA element is covered by the binding of the polymerase. For simplicity, we have indicated the initiator in only one DNA strand; however, the polymerase transcribes the other DNA strand.

tide-directed mutagenesis with the Amersham mutagenesis kit. The mutations were confirmed by sequencing through the altered regions with the Sequenase kit, as recommended by the supplier (U.S. Biochemical Corp.).

\section{Specific transcription reactions}

Reactions that measured transcription from the Ad-MLP $(40 \mu l)$ were as described previously (Flores et al. 1988) and contained $20 \mathrm{~mm}$ HEPES buffer (pH 7.9), $8 \mathrm{~mm} \mathrm{MgCl}_{2}, 60 \mathrm{mM} \mathrm{KCl}, 10 \mathrm{~mm}$ ammonium sulfate, $12 \%$ (vol/vol) glycerol, $4 \mathrm{~mm}$ dithiothreitol, 0.6 mM ribonucleoside triphosphates (ATP and CTP), $12.5 \mu \mathrm{M}\left[\alpha^{-32} \mathrm{P}\right]$ UTP $(10,000 \mathrm{cpm} / \mathrm{pmole}), 2 \%(\mathrm{wt} / \mathrm{vol})$ polyethylene glycol 8000 (PEG-8000), and DNA pML/C C $_{2} \mathrm{AT}$ ) (Sawadogo and Roeder 1985b|. Reactions were supplemented with purified transcription factors: TFIIA (single-stranded DNA agarose fraction, $0.3 \mu \mathrm{g}$ ), TFIIB (single-stranded DNA agarose fraction, 0.2 $\mu \mathrm{g}$ ), TFIID (single-stranded DNA agarose fraction, $0.2 \mu \mathrm{g}$, or as indicated in the figure legends), TFIIE/TFIIF (Affi-gel blue fraction, $0.35 \mu \mathrm{g}$ ), RNA polymerase II ( 2.8 units), and MLTF (DNA affinity step, $4 \mu \mathrm{l}$ ). Reactions that measured transcription from the adenovirus IVa2 promoter $(40 \mu \mathrm{l})$ were as described for the MLP; however, the $\mathrm{KCl}$ concentration was reduced to $45 \mathrm{mM}$ and PEG was omitted. The DNA template [plasmid DNA pAd-204, $12.5 \mu \mathrm{g} / \mathrm{ml}$ (Natarajan et al. 1984)] was linearized with the restriction endonuclease HincII. Transcription from the IVa2 major initiation site to the HincII site of plasmid pAd-204 produced an RNA molecule of 656 nucleotides. In reactions performed with circular DNA templates, the products were analyzed by primer extension. In this case, the reaction mixtures were scaled-up threefold and the primer used was complementary to residues extending from +78 to +48 on the IVa2 promoter. The factors used in the IVa2 transcription reactions were derived as follows (for details, see Reinberg et al. 1987b). TFIIA (single-stranded DNA agarose fraction, $0.8 \mu \mathrm{g}$ ), TFIIB (single-stranded DNA agarose fraction, $0.12 \mu \mathrm{g}$ ), TFIIE/TFIIF (Sephacryl S200 fraction, $1.4 \mu \mathrm{g}$ ), TFIID (carboxymethyl cellulose fraction, $0.15 \mu \mathrm{g}$, or as indicated in the figure legends), RNA polymerase II ( 2.8 units), MLTF (DNA affinity step, $4 \mu 1$ ). Transcription reactions were incubated at $30^{\circ} \mathrm{C}$ for $75 \mathrm{~min}$, unless otherwise indicated in the figure legends.

Reactions performed with nuclear extracts contained $\sim 135$ $\mu \mathrm{g}$ of protein in a 50- $\mu \mathrm{l}$ volume and were incubated for $90 \mathrm{~min}$ at $30^{\circ} \mathrm{C}$ (Dingham et al. 1983). The products of the reactions were separated by electrophoresis on $4 \%$ polyacrylamide $-7 \mathrm{M}$ urea gels.

\section{Acknowledgments}

We thank Drs. L.D. Vales, K.J. Marians, and R. Weinmann for reading the manuscript and active discussions. We also thank Dr. R.G. Roeder for providing the plasmid DNA pML $(\mathrm{C} 2 \mathrm{AT})$ and Dr. A. Berk for providing the yeast TFIID clone. The expertise of Mr. Nai-Sheng Lin in preparing the nuclear extracts is acknowledged. We also thank the members of the laboratory for active discussions. This work was supported by grants from the 
National Institutes of Health (GM 37120), the National Science Foundation (DMB 88-19342), and the New Jersey Commission on Cancer Research (687-035 and 688-026). J.C., P.C., and I.H. were supported by fellowships from Hoffman-LaRoche, Hoechst, and the core curriculum from Rutgers University, respectively. D.R. was a recipient of an American Cancer Society Junior Faculty Research Award (JFRA-205).

The publication costs of this article were defrayed in part by payment of page charges. This article must therefore be hereby marked "advertisement" in accordance with 18 USC section 1734 solely to indicate this fact.

\section{References}

Ayer, D.A. and W.S. Dynan. 1988. Simian virus 40 late promoter: A novel tripartite structure that includes intragenic sequences. Mol. Cell Biol. 8: 2021-2033.

Baker, C.C. and E.B. Ziff. 1981. Promoters and heterogeneous $5^{\prime}$ termini of the messenger RNAs of adenovirus serotype 2. $I$. Mol. Biol. 149: 189-221.

Breathnach, R. and P. Chambon. 1981. Organization and expression of eukaryotic split genes coding for proteins. Annu. Rev. Biochem. 50: 349-393.

Buratowski, S., S. Hahn, P.A. Sharp, and L. Guarente. 1988. Function of a yeast TATA element-binding protein in a mammalian transcription system. Nature 334: 37-42.

Buratowski, S., S. Hahn, L. Guarente, and P.A. Sharp. 1989. Five intermediate complexes in transcription initiation by RNA polymerase II. Cell 56: 549-561.

Carcamo, J., S. Lobos, A. Merino, L. Buckbinder, R. Weinmann, V. Natarajan, and D. Reinberg. 1989. Factors involved in specific transcription by mammalian RNA polymerase II. Role of factors IID and MLTF in transcription from the adenovirus major late and IVa2 promoters. I. Biol. Chem. 264: 7704-7714.

Carthew, R.W., L.A. Chodosh, and P.A. Sharp. 1985. An RNA polymerase II transcription factor binds to an upstream element in the adenovirus major late promoter. Cell 43: 439446.

Cavallini, B., J. Huet, J.L. Plassat, A. Sentenec, J.M. Egly, and P. Chambon. 1988. A yeast activity can substitute for the HeLa cell TATA box factor. Nature 334: 77-86.

Cavallini, B., I. Faus, H. Matthes, J.M. Chipoulet, B. Winsor, J.M. Egly, and P. Chambon. 1989. Cloning of the gene encoding the yeast protein BTF1Y, which can substitute for human TATA box-binding factor. Proc. Natl. Acad. Sci. 86: $9803-9807$.

Davidson, B.L., J.M. Egly, E.R. Mulvihill, and P. Chambon. 1983. Formation of stable preinitiation complexes between eukaryotic class B transcription factors and promoter sequences. Nature 301: 680-686.

Dignam, D.I., R.M. Lebovitz, and R.G. Roeder. 1983. Accurate transcription initiation by RNA polymerase II in a soluble extract from isolated mammalian nuclei. Nucleic Acids Res. 11: $1475-1485$.

Eisenmann, D.M., C. Dollard, and F. Winston 1989. SPT15, the gene encoding the yeast TATA binding factor TFIID, is required for normal transcription initiation in vivo. Cell 58: $1183-1191$.

Fire, A., M. Samuels, and P.A. Sharp. 1984. Interaction between RNA polymerase II, factors, and template leading to accurate transcription. I. Biol. Chem. 259: 2509-2516.

Flores, O., E. Maldonado, Z. Burton, J. Greenblatt, and D. Reinberg. 1988. Factors involved in specific transcription by mammalian RNA polymerase II. RNA polymerase II-associating protein 30 is an essential component of transcription factor IIF. I. Biol. Chem. 263: 10812-10816.

Hahn, S., S. Buratowski, P.A. Sharp, and L. Guarente. 1989. Isolation of the gene encoding the yeast TATA binding protein TFIID: A gene identical to the SPT15 suppressor of Ty element insertions. Cell 58: 1173-1181.

Horikoshi, M., M.F. Carey, H. Kakidani, and R.G. Roeder. 1988a. Mechanism of action of a yeast activator, direct effect of GAL4 derivatives on mammalian TFIID-promoter interactions. Cell 54: 665-669.

Horikoshi, M., T. Hai, Y.S. Lin, M.R. Green, and R.G. Roeder. 1988b. Transcription factor ATF interacts with the TATA factor to facilitate establishment of a preinitiation complex. Cell 54: 1033-1042.

Horikoshi, M., C.K. Wang, H. Fujii, J.A. Cromlish, P.A. Weil, and R.G. Roeder. 1989a. Cloning and structure of a yeast gene encoding a general transcription initiation factor TFIID that binds to the TATA box. Nature 341: 299-303.

. $1989 \mathrm{~b}$. Purification of a yeast TATA box-binding protein that exhibits human transcription factor IID activity. Proc. Natl. Acad. Sci. 86: 4843-4847.

Leong, K. and S.J. Flint. 1984. Specific transcription of an adenoviral gene that possesses no TATA sequence homology in extracts of HeLa cells. I. Biol. Chem. 259: 11527-11533.

McKnight, S. and R. Tjian. 1987. Transcriptional selectivity of viral genes in mammalian cells. Cell 46: 795-805.

Mermelstein, F.H., O. Flores, and D. Reinberg. 1989. Initiation of transcription by RNA polymerase II. Biochim. Biophys. Acta 1009: 1-10.

Miyamoto, N.G., V. Moncollin, M. Wintzerith, R. Hen, J.M. Egly, and P. Chambon. 1984. Stimulation of in vitro transcription by the upstream element of the adenovirus-2 major late promoter involves a specific factor. Nucleic Acids Res. 12: 8779-8799.

Miyamoto, N.G., V. Moncollin, J.M. Egly, and P. Chambon. 1985. Specific interactions between a transcription factor and the upstream element of the adenovirus- 2 major late promoter. EMBO J. 4: 3563-3570.

Moura-Neto, R., K.P. Dudor, and R. Perry. 1989. An element downstream of the CAP site is required for transcription of the gene encoding mouse ribosomal protein L 32. Proc. Natl. Acad. Sci. 86: 3997-4001.

Nakajima, N., M. Horikoshi, and R.G. Roeder. 1988. Factors involved in specific transcription by mammalian RNA polymerase II: Purification, genetic specificity and TATA boxpromoter interaction of TFIID. Mol. Cell. Biol. 8: 40284033.

Natarajan, V., M.J. Madden, and N.P. Salzman. 1984. Proximal and distal domains that control in vitro transcription of the adenovirus IVa2 gene. Proc. Natl. Acad. Sci. 81: 6290-6294. - 1985. Positive and negative control sequences within the distal domain of the adenovirus IVa2 promoter overlap with the major late promoter. J. Virol. 55: 10-15.

- 1987. Identification of a transcription factor which interacts with the distal domain of the adenovirus IVa2 promoter. J. Virol. 61: 646-652.

Reinberg, D. and R.G. Roeder. 1987. Factors involved in specific transcription by mammalian RNA polymerase II. Purification and functional analysis of initiation factors IIB and IIE. I. Biol. Chem. 262: 3310-3321.

Reinberg, D., O. Flores, and L. Buckbinder. 1987a. Characterization and functional analysis of the factors required for transcription of the Adenovirus Major Late Promoter. In Molecular biology of RNA: New perspectives (ed. M. Inouye and B. Dudock), pp. 423-439. Academic Press, California.

Reinberg, D., M. Horikoshi, and R.G. Roeder. 1987b. Factors involved in specific transcription by mammalian RNA poly- 
Carcamo et al.

merase II. Functional analysis of initiation factors IIA and IID and identification of a new factor operating at sequences downstream of the initiation site. J. Biol. Chem. 262: 33223330.

Saltzman, A.G. and R. Weinmann. 1989. Promoter specificity and modulation of RNA polymerase II transcription. FASEB J. 3: $1723-1728$.

Samuels, M., A. Fire, and P.A. Sharp. 1982. Separation and characterization of factors mediating accurate transcription by RNA polymerase II. J. Biol. Chem. 257: 14419-14427.

Sawadogo, M. and R.G. Roeder. 1985a. Interaction of a genespecific transcription factor with the adenovirus major late promoter upstream of the TATA box region. Cell 43: 165175.

. 1985b. Factors involved in specific transcription by human RNA polymerase II. Analysis by a rapid and quantitative in vitro assay. Proc. Natl. Acad. Sci. 82: 4394-4398.

Schmidt, M.C., C. Kao, R. Pei, and A. Berk. 1989. Yeast TATAbox transcription factor gene. Proc. Natl. Acad. Sci. 86: 7785-7789.

Smale, T.S. and D. Baltimore. 1989. The initiator as a transcriptional control element. Cell 57: 103-113.

VanDyke, M.W., R.G. Roeder, and M. Sawadogo. 1988. Physical analysis of transcription preinitiation complex assembly on class II gene promoter. Science 241: 1335-1338.

Widen, S.G., P. Kedar, and S.H. Wilson. 1988. Human b-polymerase gene. Structure of the 5 -flanking region and active promoter. J. Biol. Chem. 264: 16992-16998.

Yu, Y.-T. and J.L. Manly. 1986. Structure and function of the S1 nuclease-sensitive site in the adenovirus late promoter. Cell 45: $743-751$. 


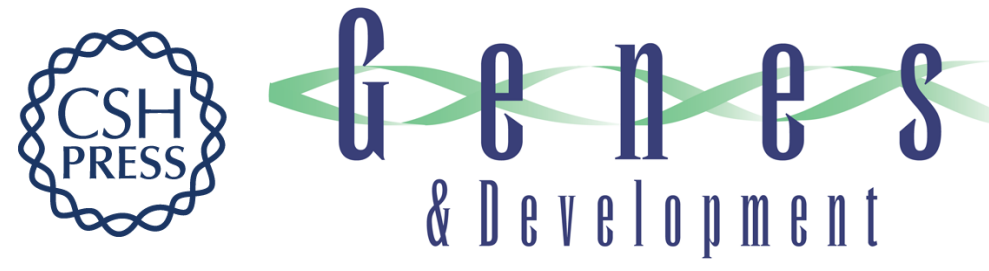

\section{A TATA-like sequence located downstream of the transcription initiation site is required for expression of an RNA polymerase II transcribed gene.}

J Carcamo, E Maldonado, P Cortes, et al.

Genes Dev. 1990, 4:

Access the most recent version at doi:10.1101/gad.4.9.1611

References This article cites 40 articles, 18 of which can be accessed free at: http://genesdev.cshlp.org/content/4/9/1611.full.html\#ref-list-1

License

Email Alerting Service

Receive free email alerts when new articles cite this article - sign up in the box at the top right corner of the article or click here.

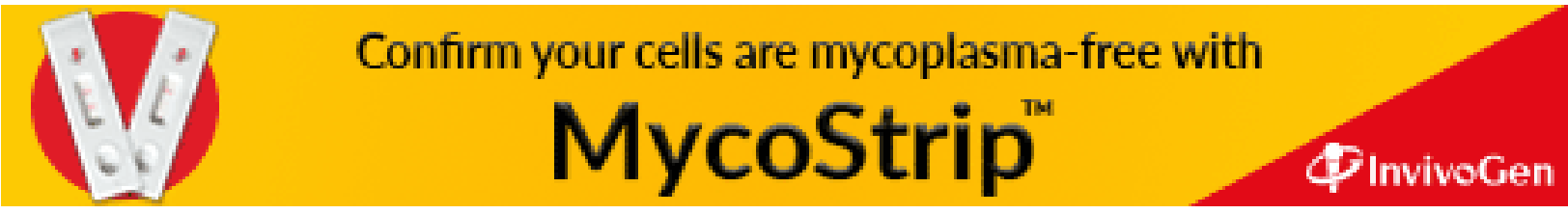

